\title{
Determination of Chorionicity in Twins: Feasibility at a Tertiary Care Centre and Audit of Current Practice in the Community
}

\author{
M. L. Pilli • S. L. Balusamy • J. Ramesh • \\ I. Suresh $\cdot$ S. Seshadri $\cdot$ S. Sairam
}

Received: 30 April 2014/Accepted: 21 May 2014/Published online: 3 July 2014

(C) Society of Fetal Medicine 2014

\begin{abstract}
To assess the feasibility and to audit the practice of determining chorionicity in twin pregnancies in South India. Sonographic findings from first trimester scans of twin pregnancies were retrospectively analysed to evaluate the feasibility of determining chorionicity in the first visit to the unit. In addition, an audit was carried out on all twin pregnancies referred to the authors in the second trimester to ascertain whether chorionicity had been established prior to referral. It was feasible to determine chorionicity at the first visit in $100 \%$ of the 611 twin pregnancies that were seen at 11-14 weeks in the unit. However, the audit revealed that only $40 \%$ of the referred population had a diagnosis of chorionicity prior to referral. Whilst it is feasible to determine chorionicity in all twin pregnancies in the first trimester, the practice is not widespread in the referring community in South India and this needs to be addressed.
\end{abstract}

Keywords Multiple pregnancy - Twins - Chorionicity · Monochorionic $\cdot$ Dichorionic $\cdot$ First trimester

\section{Introduction}

The identification of chorionicity in a twin pregnancy is essential for effective screening for aneuploidies, prenatal diagnosis, and in the management of twin pregnancies discordant for anomalies, growth restriction and intrauterine

M. L. Pilli · S. L. Balusamy $(\varangle) \cdot$ J. Ramesh · I. Suresh .

S. Seshadri $\cdot$ S. Sairam

First Trimester Screening Unit, Department of Fetal Medicine,

Mediscan Systems, 197, Natesan Road, Mylapore,

Chennai 600004, Tamil Nadu, India

e-mail: sbsatthya@yahoo.com death [1]. Early detection of chorionicity allows stratification of risk in twins and promotes organisation of appropriate monitoring and surveillance. This enables the diagnosis of specific complications such as twin-to-twin transfusion syndrome (TTTS) at an early stage and provides treatment options at the appropriate gestation [2].

Chorionicity can be determined with $100 \%$ accuracy by demonstrating the twin-peak sign (lambda sign) or the T-sign on ultrasound at 11-14 weeks to identify dichorionicity and monochorionicity respectively. The lambda sign is thought to be due to the presence of chorionic tissue in the inter-twin membrane at the point of its attachment to the placenta. As gestation advances, there is a likelihood of increasing regression of this tissue. Sepulveda et al. [3] have demonstrated that the lambda sign disappears in about $7 \%$ of dichorionic twins with fused placentae, between 14 and 20 weeks of pregnancy. It can therefore become difficult to establish chorionicity reliably after 14 weeks. In monochorionic twin pregnancies, absence of proliferation of chorionic tissue between the inter-twin membranes gives rise to T-sign.

The aim of this study was to assess the feasibility of identification of the chorionicity during the 11-14 weeks scan in a tertiary referral center in South India. We also carried out an audit of the current practice of determining chorionicity in the first trimester in the referring community.

\section{Materials and Methods}

This was a retrospective study analyzing data from January 2009 to December 2010. All twin pregnancies seen in the First Trimester Screening Unit (FTSU) during the study period were reviewed. Only those with two viable fetuses 


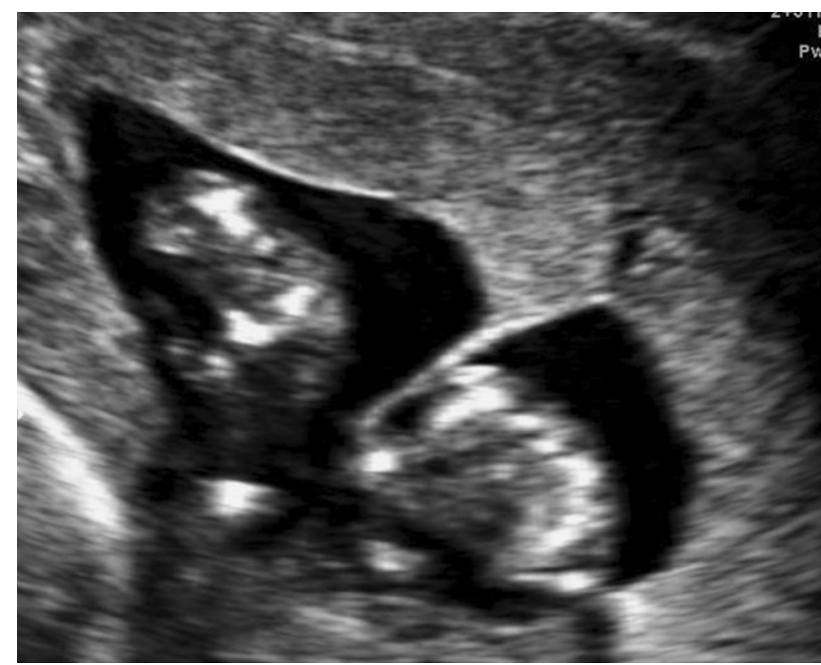

Fig. 1 Ultrasound image of lambda sign in first trimester

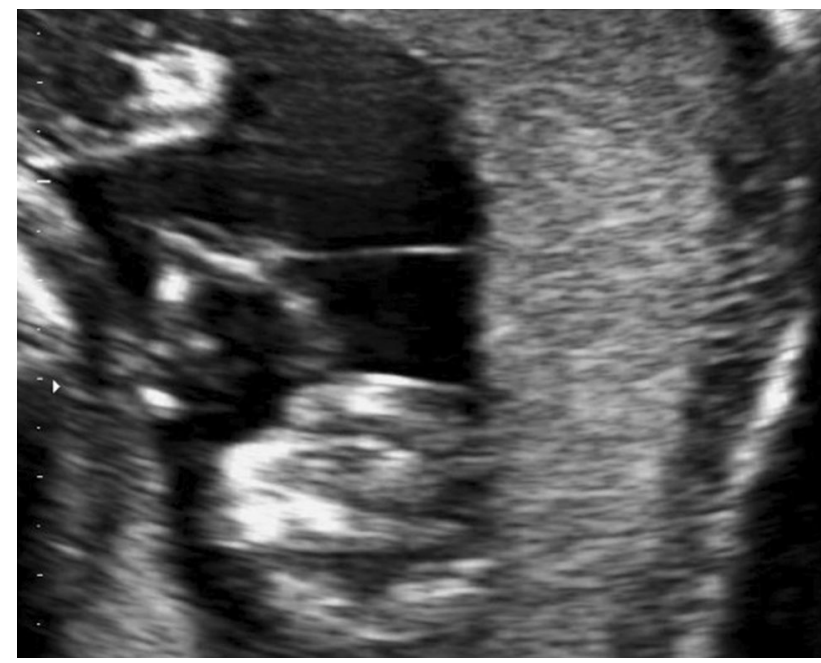

Fig. 2 Ultrasound image of T-sign in first trimester

at the time of the 11-14 weeks scan were included in the study.

In the unit, images and video clips are archived prospectively in a database (Sonocare, Medialogic Solutions, India Pvt Ltd.) and can be retrieved for analysis. Two reviewers jointly reviewed the ultrasound findings that had been recorded during the scan carried out on the study group. The still images and video clips were screened to evaluate whether two separate placental masses were seen and when a single placental mass was seen, whether a lambda (Fig. 1) or T-sign (Fig. 2) had been demonstrated and whether this had been possible in the first visit.

All scans had been carried out on two machines (Philips HD 15, Japan, Voluson E8, GE Healthcare systems, Germany) by trained sonographers.

For the audit, all cases of twin pregnancies referred for the first time in the second trimester to the center from

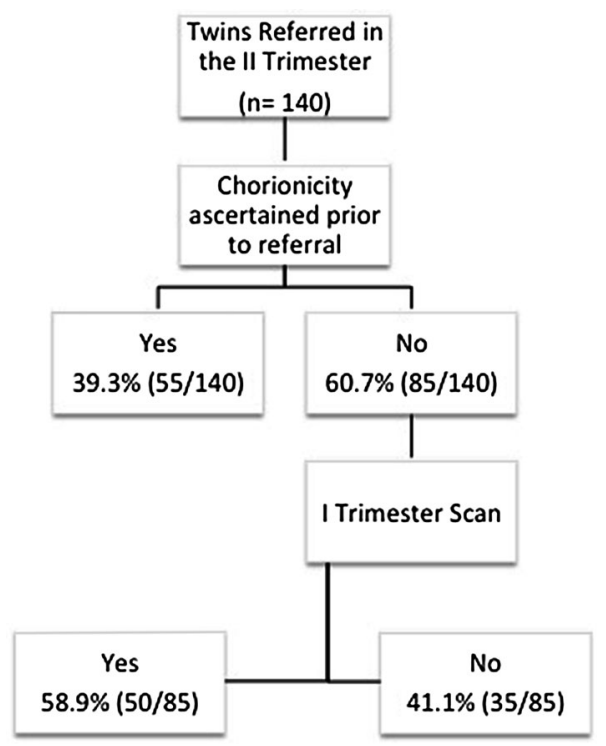

Fig. 3 Flow chart showing audit results

January 2010 to September 2010, were reviewed. The case files were examined to verify whether a first trimester ultrasound scan had been performed and whether any attempt at establishing chorionicity had been made.

\section{Results}

Scan in the FTSU was done in 611 twin pregnancies with two viable fetuses at 11-14 weeks during the study period. The mean gestational age at the time of the scan was 12 weeks 4 days ( $S D \pm 6$ days). Data regarding mode of conception was available in 516 pregnancies. Of these, $67.8 \%(350 / 516)$ were as a result of assisted reproductive technology (ART) and $32.2 \%(166 / 516)$ were natural conceptions.

Chorionicity had been established in all these pregnancies in the first visit. Five pregnancies had been diagnosed as monochorionic-monoamniotic twins with no demonstrable inter-twin membrane. In 606 twins, two separate placental masses or a lambda or T-sign had been recorded. Out of these, $85.9 \%(521 / 606)$ were dichorionic-diamniotic twins and $14.1 \%(85 / 606)$ were monochorionicdiamniotic twins. Monochorionic twins formed 5.2 and $30.1 \%$ of the ART and natural pregnancies respectively.

A total of 140 twin pregnancies were referred to us for the first time in the second trimester during the study period. Out of these, $40 \%(55 / 140)$ had a diagnosis of chorionicity prior to referral. In the remaining $60 \%(85 /$ 140) chorionicity was not determined although, $58.8 \%$ (50/85) of them had undergone a first trimester scan (Fig. 3). The mean gestational age at referral was 20 weeks and 6 days ( $\mathrm{SD} \pm 2.1$ weeks). 
Overall, $75 \%(105 / 140)$ of the referred twin pregnancies had undergone a first trimester scan with $49.5 \%$ (52/ $105)$ of the scans being at 11-14 weeks gestation. A significant proportion of these twin pregnancies [41.2 \% (58/ 140)] had been conceived with the help of ART.

\section{Discussion}

This study demonstrates that it is feasible to determine chorionicity in $100 \%$ of twin pregnancies at the 11-14 weeks scan in a tertiary referral center in South India. It has also shown that $60 \%$ of the referred twin pregnancies in the region did not have this diagnosis. This is despite a significant proportion of them having a first trimester scan $(75 \%)$.

The findings in the present feasibility study are comparable to that of previous studies [3-6]. In a prospective study conducted by Caroll et al. [4], it was $100 \%$ feasible to determine chorionicity in the entire 150 twin pregnancies scanned at 10-14 weeks, with $99.3 \%$ accuracy. Sepulveda et al. also demonstrate $100 \%$ feasibility in the determination of chorionicity with $100 \%$ accuracy. Validation of the scan findings were done with placental histology in the former and with gender of the babies in the latter study and with a combination of the two in the last one. In the current study, validation was not possible with either method.

The audit carried out on the referred cases demonstrated that only $40 \%$ of these had a diagnosis of chorionicity. These cases were referred to the authors from a mixture of clinics that included fertility centers, diagnostic centers and peripheral obstetric practices. A recent French study addressed the same issue and found that of the 31 twin pregnancies referred to their unit, only $77 \%$ had a diagnosis of chorionicity [7]. A low rate of diagnosis of the chorionicity in the referred group in the current study is likely to be due to a combination of factors such as:

1. Lack of knowledge about chorionicity and its significance, both on the part of the Obstetrician requesting the scan and the technician performing the scan.

2. Lack of adequate training.

3. Patients not being aware of the need for determining chorionicity.

This highlights the need for improving the awareness about the need for determination of chorionicity, so that it can be addressed as early in the pregnancy as possible. A significant proportion of these twins' pregnancies [41.2\% $(58 / 140)]$ had been conceived with the help of ART that were likely to have had several early scans. Of these only $29.3 \%(17 / 58)$ had a diagnosis of chorionicity. Details of all early scans were not available to the authors and hence it was not possible to assess the average number of scans in these pregnancies.

Chorionicity can be determined as early as 6-9 weeks in the first trimester. It can be reliably assessed at the 11-14 weeks scan, when the pregnancy can also be screened for chromosomal, cardiac, and structural abnormalities. In twins, this $11-14$ weeks scan is crucial, as discrepancy in nuchal translucency between twins and abnormal flow in the ductus venosus are considered as pointers for evolving TTTS [8, 9]. In most developed countries, the 11-14 weeks scan is becoming standard practice for both singleton and twin pregnancies [10]. Additionally, as the lambda sign cannot be used reliably in the second trimester, it is important that a clear documentation of the chorionicity is made before 14 weeks, ideally as part of the 11-14 weeks scan.

India is divided into 29 states and seven union territories. Tamil Nadu (TN) is one of the states and holds a population of 72 million based on the recent census data (Source: http://censusindia.gov.in/). Tamil Nadu is divided into 32 districts, with the capital city being Chennai. The district of Chennai alone holds 4.6 million people $(6.5 \%$ of the population of Tamil Nadu, Source: http://censusindia. gov.in/) and houses 384 private ultrasound scan centers as of 2002 (Source: www.tnhealth.org/scanlist.txt). This number is likely to be higher in the recent years. In addition, the Indian government has instituted the National Rural Health Mission (NRHM) Project, which amongst many other issues, also addresses the need to provide ultrasound equipment and training at a basic level to government doctors working in the rural areas in TN. There is therefore, no dearth of resources for provision of access to ultrasound scans for all pregnant women. This means that all strata of pregnant women will have access to ultrasound scans during their pregnancy. At this point of health-system development, such audits would be of use in establishing appropriate guidelines as the training is planned and executed. The authors are involved directly in arranging these training sessions. They intend to capitalize on these opportunities to promote awareness about the role of chorionicity and the feasibility of identifying this at the 11-14 weeks scan in twins.

The increasing demand for ART has resulted in mushrooming of infertility clinics in India. There is no reliable information on the number of ART clinics in India in the absence of a national registry of ART clinics [11]. In the audit study the authors found that $41.2 \%(58 / 140)$ of the referred pregnancies were conceived by ART. Of these, chorionicity had been determined in only $29.3 \%(17 / 58)$ in spite of multiple early pregnancy scans. Copperman et al. [12] have already addressed this and demonstrated $100 \%$ accuracy in determining chorionicity in a small group of 31 IVF pregnancies, that underwent early transvaginal scans 
in the first trimester. Infertility centers need to be made aware of the pivotal role of chorionicity in the management of twin pregnancies and the need to document this when they have the opportunity to scan these pregnancies at a very early gestation.

Roland et al. have shown that after a basic training for a minimum of 6 months on ultrasonographic characteristics of MC and DC pregnancies, the less experienced sonographers could determine chorionicity as accurately as the experienced sonographers [13]. This emphasizes on the need for basic knowledge and adequate training before performing obstetric scans. Weisz et al. [14] have demonstrated that sonographers and fetal medicine specialists concur $90 \%$ of the times when making a diagnosis of chorionicity. The rate of discordant results was $5.5 \%$ for monochorionic-diamniotic pregnancies and $0.8 \%$ for dichorionic pregnancies. This discrepancy can be overcome by referring all suspected cases of monochorionicity to a specialist unit that can confirm the chorionicity and also draw out appropriate follow up plans for management of this high-risk twin pregnancy. This concept of triaging the twin pregnancies at the 11-14 weeks scan is especially relevant in early identification of complications in monochorionic pregnancies. The center is in a position to offer fetoscopic procedures as treatment options in India and can therefore effectively treat these complications.

The major limitation of the present study is the lack of validation. It addresses the feasibility, but not the accuracy of the determination of chorionicity. This is because, confirmatory examination of the placentae or DNA studies are not feasible on every patient owing to the cost factor. Secondly, acquiring information regarding outcome of pregnancy is an arduous task in this cohort as they are referred from various centers that do not maintain outcome records and often deal with a migratory population. Despite this, it has highlighted the need for creating awareness about chorionicity amongst practitioners and the patients.

\section{Conclusions}

According to the authors, this is probably the first report on determination of chorionicity in Southern India. It has highlighted the lack of consistency in identifying chorionicity, a key finding, amongst practitioners in the referring community who feed cases to the tertiary center. It is disappointing that when it is $100 \%$ feasible to determine the chorionicity in the first trimester, it is still not a universal practice, in our region. Timing the scan between 11 and 14 weeks period of gestation in twin pregnancies is ideal for assessing chorionicity along with nuchal translucency and ductus venosus, to screen for aneuploidy and TTTS. The authors believe that this paper would be the first step in creating management guidelines for twin pregnancies in India, and more importantly in creating awareness amongst practitioners and prospective parents. Based on their findings, the authors would recommend that every center (including infertility centers) that offers obstetric scans be aware of and be trained to identify chorionicity in twins. Further, the authors would recommend that when the chorionicity is not clear at the 11-14 weeks scan, these cases are referred to an experienced tertiary center.

\section{Conflict of Interest None.}

\section{References}

1. Bhide A, Thilaganathan B. What prenatal diagnosis should be offered in multiple pregnancy? Best Pract Res Clin Obstet Gynaecol. 2004;18(4):531-42.

2. Allen MH, Garabelis NS, Bornick PW, Quintero RA. Minimally invasive treatment of twin-to-twin transfusion syndrome. AORN J. 2000;71(4):796, 801-10; quiz 811-2, 815-8.

3. Sepulveda W, Sebire NJ, Hughes K, Kalogeropoulos A, Nicolaides $\mathrm{KH}$. Evolution of the lambda or twin-chorionic peak sign in dichorionic twin pregnancies. Obstet Gynecol. 1997;89(3):439-41.

4. Carroll SG, Soothill PW, Abdel-Fattah SA, Porter H, Montague I, Kyle PM. Prediction of chorionicity in twin pregnancies at 10-14 weeks of gestation. BJOG. 2002;109(2):182-6.

5. Finberg HJ. The 'twin peak' sign: reliable evidence of dichorionic twinning. J Ultrasound Med. 1992;11(11):571-7.

6. Dias T, Arcangeli T, Bhide A, Napolitano R, Mahsud-Dornan S, Thilaganathan B. First-trimester ultrasound determination of chorionicity in twin pregnancy. Ultrasound Obstet Gynecol. 2011;38(5):530-2.

7. Levy R, Arfi JS, Mirlesse V, Jacob D. Ultrasonic diagnosis of chorionicity in multiple pregnancies. Gynecol Obstet Fertil. 2003;31(11):960-3.

8. Nicolaides KH. Nuchal translucency and other first-trimester sonographic markers of chromosomal abnormalities. Am J Obstet Gynecol. 2004;191(1):45-67.

9. Matias A, Montenegro N, Loureiro T, Cunha M, Duarte S, Freitas D, Severo M. Screening for twin-twin transfusion syndrome at 11-14 weeks of pregnancy: the key role of ductus venosus blood flow assessment. Ultrasound Obstet Gynecol. 2010;35(2):142-8.

10. NICE. CG62 - antenatal care: routine care for the healthy pregnant woman. London: National Institute for Clinical Excellence; 2008.

11. National Guidelines for Accreditation, Supervision and Regulation of ART Clinics in India. Indian Council of Medical Research National Academy of Medical Sciences (India), New Delhi, India.

12. Copperman AB, Kaltenbacher L, Walker B, Sandler B, Bustillo M, Grunfeld L. Early first trimester ultrasound provides a window through which the chorionicity of twins can be diagnosed in an in vitro fertilization (IVF) population. J Assist Reprod Genet. 1995;12(10):693-7.

13. Devlieger RGL, Demeyere T, Deprest JA, Schoubroeck DV, Witters I. Ultrasound determination of chorionicity in twin pregnancy: accuracy and operator experience. Twin Res. 2001;4(4):223-6.

14. Weisz B, Pandya P, Dave R, Jauniaux E. Scanning for chorionicity: comparison between sonographers and perinatologists. Prenat Diagn. 2005;25(9):835-8. 